\title{
CLUSTERING ALGORITHM FOR DETECTION IN THE DISCRETE NONLINEAR FOURIER SPECTRUM Benedikt Leible ${ }^{*}$, Francisco Javier García-Gómez ${ }^{1}$, Norbert Hanik ${ }^{1}$
}

\author{
${ }^{1}$ Institute for Communications Engineering, Technical University Munich, Munich, Germany \\ *benedikt.leible@tum.de
}

\begin{abstract}
Keywords: NONLINEAR OPTICS, CLUSTERING METHODS, NONLINEAR DISTORTION, OPTICAL FIBER COMMUNICATION, OPTICAL SOLITONS
\end{abstract}

\begin{abstract}
\section{Introduction}

For state-of-the-art high-data-rate transmission over optical fiber, nonlinearity limits the achievable rates in the high power regime [1]. With an ever growing demand for increasing data rates, the question arises whether the capacity decrease in the high power regime is something inherent to the fiber channel, or can be overcome by switching the domain in which the signal is modulated [2].
\end{abstract}

Expectation maximization clustering of received nonlinear spectral data from solitonic pulses is used to reduce bit error rates. Simulations over a $1800 \mathrm{~km}$ Raman amplified fiber link demonstrate the effectiveness of the method.

The nonlinear Fourier transform (NFT) [2, 3] was suggested as a promising tool to overcome these limitations, since it incorporates dispersion and nonlinearity into the transmission instead of treating them as impairments. The NFT was conceived for the deterministic lossless nonlinear Schrödinger equation (NLSE), and the impact of fiber loss and distributed noise on the nonlinear Fourier domain is not yet very well quantified, though some results have been obtained using perturbation theory $[4,5]$. In [6], a time-domain pulse with circularly-symmetric additive white Gaussian noise is considered. It is shown that, in a first-order approximation, the noise in the nonlinear spectral domain stays Gaussian but it is not necessarily circularly symmetric or white.

This led us to the idea of using the expectation maximization (EM) algorithm [7] to obtain the statistics of the received symbols in the nonlinear Fourier domain, improving the detection performance. A similar approach was presented in [8] to jointly mitigate impairments due to fiber nonlinearity, I/Q-imbalance and laser linewidth in a linearly modulated 16-QAM dual polarization optical transmission setup. We adapt this approach for improved detection directly in the nonlinear Fourier domain. Similar to [8], our approach is blind and does not require pilot symbols to estimate the channel properties.

In the following, we introduce the NFT, the principle of Raman amplification, and the EM algorithm. We then present simulation results that validate our approach for an optical fiber channel with non-ideal Raman amplification and distributed ASE noise.

\section{The Nonlinear Fourier Transform}

Consider the normalized nonlinear Schrödinger equation [2]

$$
\frac{\partial q(t, z)}{\partial z}=\mathrm{j} \frac{\partial^{2} q(t, z)}{\partial t^{2}}+\mathrm{j} 2|q(t, z)|^{2} q(t, z)
$$

where $q(t, z), t$ and $z$ are the normalized complex signal, normalized time and normalized propagation distance respectively, while $\mathrm{j}$ represents the imaginary unit.

The NFT and its inverse (INFT) enable the modulation of data onto the nonlinear Fourier spectra [2]. Each time domain signal $q(t, z)$ can be represented by its two corresponding nonlinear spectra, which are obtained from the nonlinear Fourier coefficients $a(\lambda)$ and $b(\lambda)$ computed by the NFT.

The continuous spectrum $q_{\mathrm{c}}(\lambda)=b(\lambda) / a(\lambda)$ is defined along the real line $(\lambda \in \mathbb{R})$ of the generalized frequency support $\lambda \in \mathbb{C}$ and can be seen as an analog to the linear frequency spectrum of the standard Fourier transform. The discrete spectrum, which is defined only for a finite number of eigenvalues with a positive imaginary part $\left(\lambda_{k} \in \mathbb{C}^{+}\right)$, has no analog in linear channels. These discrete generalized frequencies $\lambda_{k}$, together with the corresponding complex nonlinear Fourier coefficients $b_{k} \equiv b\left(\lambda_{k}\right)$, define the discrete spectrum, which corresponds to the solitonic part of the signal and is used in this paper exclusively $\left(q_{\mathrm{c}}(\lambda)=0\right)$.

This representation of the signal has the benefit that even though the corresponding time domain signal $q(t, z)$ propagates according to the NLSE in a complicated manner, the values $\lambda_{k}$ do not change during propagation and the spectral amplitudes $q_{c}(\lambda)$ and $b_{k}$ are independent of each other for each generalized frequency.

The influence of signal propagation along the fiber in the nonlinear Fourier domain is given by

$$
\frac{q_{\mathrm{c}}(\lambda, z)}{q_{\mathrm{c}}(\lambda, 0)}=\mathrm{e}^{4 \mathrm{j} \lambda^{2} z}, \quad \frac{b_{k}(z)}{b_{k}(0)}=\mathrm{e}^{4 \mathrm{j} \lambda_{k}^{2} z} .
$$

\section{Transmission over Raman-Amplified Fiber}

The channel model used in this paper is the non-ideal Raman amplified single-mode fiber [9] with one co- and one counterpropagating pump laser per span: 


$$
\begin{aligned}
\frac{\partial A}{\partial Z} & =\frac{1}{2}\left[-\alpha+g_{\mathrm{R}} P_{\mathrm{p}}(Z)\right] A-\mathrm{j} \frac{\beta_{2}}{2} \frac{\partial^{2} A}{\partial T^{2}} \\
& +\mathrm{j} \gamma\left[|A|^{2}+\left(2-f_{\mathrm{R}}\right) P_{\mathrm{p}}(Z)\right] A+N(Z, T)
\end{aligned}
$$

where

$$
P_{\mathrm{p}}(Z)=P_{\mathrm{p} 0}\left(\mathrm{e}^{-\alpha_{\mathrm{p}} Z}+\mathrm{e}^{-\alpha_{\mathrm{p}}\left(\mathcal{L}_{\mathrm{s}}-Z\right)}\right)
$$

is the distance-dependent pump power. $A, Z$ and $T$ are the (unnormalized) complex signal, transmission distance and time respectively. $N(Z, T)$ is the distributed noise, whose statistics is provided in [9]. The parameters $\alpha, \beta_{2}$ and $\gamma$ are the signal attenuation, chromatic dispersion and nonlinearity coefficients, respectively. The attenuation at the wavelength of the pump laser is given by $\alpha_{\mathrm{p}}$, and $\mathcal{L}_{\mathrm{s}}$ is the length of one fiber span. The Raman gain coefficient $g_{\mathrm{R}}$ is frequency-dependent and was computed according to [10]. The factor $f_{\mathrm{R}}$ stems from the delayed Raman response of the fiber and is set to the value of 0.18 for standard single mode fibers [9].

Realistic Raman amplification not only incorporates distributed noise into the simulation, but it also models partial residual fiber loss and over-amplification along one fiber span. The pump powers of the lasers were both set to

$$
P_{\mathrm{p} 0}=\frac{1}{2} \frac{\alpha \alpha_{\mathrm{p}} \mathcal{L}_{\mathrm{s}}}{g_{\mathrm{R}}\left(1-\mathrm{e}^{-\alpha_{\mathrm{p}} \mathcal{L}_{\mathrm{s}}}\right)}
$$

such that the attenuation of the signal due to fiber loss is compensated at the end of each span. In Fig. 1, the effective Raman gain and the resulting signal power are shown for $\mathcal{L}_{\mathrm{s}}=25 \mathrm{~km}$. Further parameters are given in Table 1.

\section{Clustering by Expectation Maximization}

Consider an NFT communications system that transmits $N$ symbols of information on the spectral amplitudes $b_{k}$ of a solitonic pulse with $K$ eigenvalues. Assuming that each $b_{k}$ is taken from a constellation of $Q$ points, there are $M=Q^{K}$ possible transmit symbols, or constellation points

$$
\boldsymbol{b}_{m}=\left(\Re b_{1}[m], \ldots, \Re b_{K}[m], \Im b_{1}[m], \ldots, \Im b_{K}[m]\right)^{\mathrm{T}}
$$

for $m \in\{1, \ldots, M\}$, where $\boldsymbol{a}^{\mathrm{T}}$ denotes the transpose of $\boldsymbol{a}$. At each time index $n \in\{1, \ldots, N\}$, one constellation point $\boldsymbol{b}_{m}$ is chosen, and the symbol

$$
\begin{aligned}
\boldsymbol{x}_{n} & =\boldsymbol{b}_{m} \\
& =\left(\Re x_{1}[n], \ldots, \Re x_{K}[n], \mathfrak{\Im} x_{1}[n], \ldots, \mathfrak{I} x_{K}[n]\right)^{\mathrm{T}}
\end{aligned}
$$

is transmitted, where $\mathfrak{R}$ and $\mathfrak{J}$ denote real and imaginary parts respectively.

Let $\boldsymbol{y}_{n}$ be the received symbol at time index $n$. It can be modeled as a random variable (r.v.) $\boldsymbol{y}$ whose probability density function (PDF) is a mixture of Gaussians (MOG)

$$
p_{\boldsymbol{y}}(\boldsymbol{y})=\sum_{m=1}^{M} P_{m}(m) \mathrm{N}\left(\boldsymbol{y} \mid \boldsymbol{\mu}_{m}, \boldsymbol{\Sigma}_{m}\right)
$$

where $P_{m}(m)$ is the probability that the $m$-th constellation point $\boldsymbol{b}_{m}$ has been sent, equal to $1 / M$ for uniformly distributed constellation points. The vector $\boldsymbol{\mu}_{m}=\mathbb{E}\left[\boldsymbol{y} \mid \boldsymbol{x}=\boldsymbol{b}_{m}\right]$ is the expectation of the received symbol given that the constellation point $\boldsymbol{b}_{m}$ was sent, and $\boldsymbol{\Sigma}_{m}$ is the corresponding covariance matrix. Further, $\mathrm{N}\left(\boldsymbol{y} \mid \boldsymbol{\mu}_{m}, \boldsymbol{\Sigma}_{m}\right)$ is the multivariate Gaussian PDF with mean $\boldsymbol{\mu}_{m}$ and covariance matrix $\boldsymbol{\Sigma}_{m}$.

If a sufficient amount of received constellation points is available, the parameters of the MOG model can be estimated by the EM algorithm. EM [7] is an iterative algorithm to find a set of parameters $\Xi_{\text {opt }}=\left(\boldsymbol{\mu}_{m}, \boldsymbol{\Sigma}_{m}\right)$ (which contains all the $\boldsymbol{\mu}_{m}$ and $\boldsymbol{\Sigma}_{m}$ for $\left.m=\{1, \ldots, M\}\right)$ that maximizes the probability of the received signal $\boldsymbol{Y}=\left(\boldsymbol{y}_{1}, \ldots, \boldsymbol{y}_{N}\right)$. Thus, the estimation problem is

$$
\Xi_{\text {sol }}=\underset{\Xi}{\operatorname{argmax}}(p(\boldsymbol{Y} \mid \Xi))
$$

with $\Xi$ being the set of parameters over which we maximize. The $l$-th iteration of EM is comprised of an expectation step and a maximization step.

\subsection{Expectation Step}

For each received constellation point $\boldsymbol{y}_{n}$, the expectation step computes the a posteriori probabilities

$$
\begin{aligned}
\gamma_{n m}^{(l)} & \equiv p\left(\boldsymbol{x}_{n}=\boldsymbol{b}_{m} \mid \boldsymbol{y}_{n}\right) \\
& =\frac{P_{m}^{(l-1)}(m) \mathrm{N}\left(\boldsymbol{y}_{m} \mid \boldsymbol{\mu}_{m}^{(l-1)}, \boldsymbol{\Sigma}_{m}^{(l-1)}\right)}{\sum_{m^{\prime}=1}^{M} P_{m}^{(l-1)}\left(m^{\prime}\right) \mathrm{N}\left(\boldsymbol{y}_{n} \mid \boldsymbol{\mu}_{m^{\prime}}^{(l-1)}, \mathbf{\Sigma}_{m^{\prime}}^{(l-1)}\right)}
\end{aligned}
$$

which are often called responsibilities [8]. The superscript $a^{(l)}$ denotes the value of $a$ at the $l$-th iteration.

\subsection{Maximization Step}

In the subsequent maximization step, the parameters $\Xi^{(l)}$ are updated for all $m$ according to

$$
\begin{gathered}
N_{m}^{(l)}=\sum_{n=1}^{N} \gamma_{n m}^{(l)} \\
P_{m}^{(l)}(m)=\frac{N_{m}^{(l)}}{N} \\
\boldsymbol{\mu}_{m}^{(l)}=\frac{1}{N_{m}^{(l)}} \sum_{n=1}^{N} \gamma_{n m}^{(l)} \boldsymbol{y}_{n} \\
\boldsymbol{\Sigma}_{m}^{(l)}=\frac{1}{N_{m}^{(l)}} \sum_{n=1}^{N} \gamma_{n m}^{(l)}\left(\boldsymbol{y}_{n}-\boldsymbol{\mu}_{m}^{(l)}\right)\left(\boldsymbol{y}_{n}-\boldsymbol{\mu}_{m}^{(l)}\right)^{\mathrm{T}} .
\end{gathered}
$$

The parameters computed in the maximization step are then fed back to the next expectation step during iteration $l+1$. Note that the algorithm also estimates the input distribution $P_{m}(m)$, and is therefore also useful for non-uniform input distributions. The algorithm terminates if either an appropriate difference measure between subsequently computed parameter sets is smaller than some threshold value or after a maximum number of iterations has been reached. The EM algorithm is guaranteed to converge to a (local) maximum [8].

The EM algorithm classifies the received symbols into $M$ clusters, which correspond to the possible constellation points. The task of assigning the clusters to the constellation points is an instance of the assignment problem, and we solved it using the optimal Hungarian method [11]. This method finds an assignment $f:\{1, \ldots, M\} \rightarrow\{1, \ldots, M\}$ that minimizes the sum of Euclidean distances between the cluster means $\boldsymbol{\mu}_{m}$ and their corresponding constellation point $\boldsymbol{b}_{f(m)}$. 


\section{$5 \quad$ Numerical Results}

Table 1 Simulation parameters

\begin{tabular}{cc}
\hline Parameter & Value \\
\hline Dispersion coefficient & $\beta_{2}=-21.683 \mathrm{ps}^{2} / \mathrm{km}$ \\
Nonlinear coefficient & $\gamma=1.3174 \mathrm{~W}^{-1} \mathrm{~km}^{-1}$ \\
Signal wavelength & $\lambda=1550 \mathrm{~nm}$ \\
Attenuation at $\lambda$ & $\alpha=0.2 \mathrm{~dB} / \mathrm{km}$ \\
Pump wavelength & $\lambda_{\mathrm{p}}=1452.50 \mathrm{~nm}$ \\
Attenuation at $\lambda_{\mathrm{p}}$ & $\alpha_{\mathrm{p}}=0.25 \mathrm{~dB} / \mathrm{km}$ \\
\hline
\end{tabular}

We simulated the NFT communications system depicted in Fig. 2 over a $1800 \mathrm{~km}$ link using non-ideal distributed Raman amplification with the parameters in Table 1. The simulated link had 60 spans of $25 \mathrm{~km}$, with forward and backward Raman pumps in each span (pump power $P_{\mathrm{p} 0}=18.095 \mathrm{dBm}$ ). The Raman gain and signal power profiles along the first span are plotted in Fig. 1.
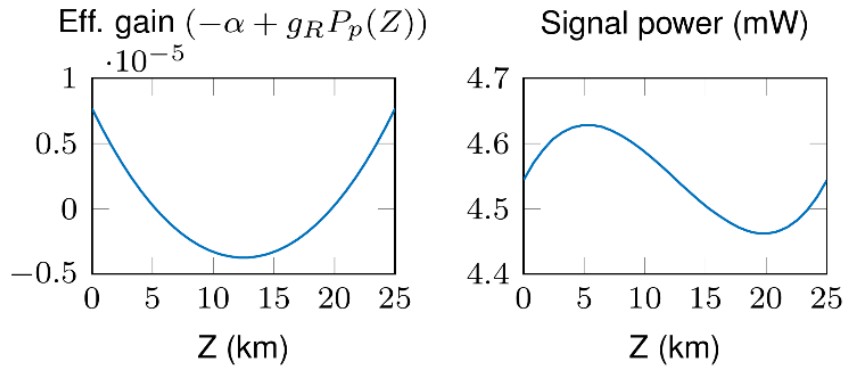

Fig. 1 Effective gain (left) and signal power (right) along a 25 km span with bi-directionally pumped Raman amplification.

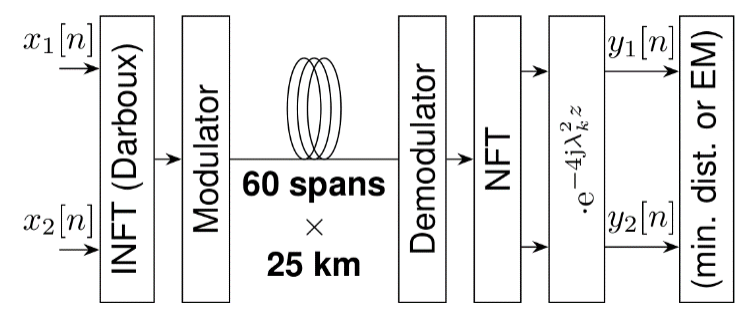

Fig. 2: Simulation setup.

In our simulation, we used 2-soliton pulses $(K=2)$ with eigenvalues $\lambda_{1}=1.5 \mathrm{j}$ and $\lambda_{2}=1 \mathrm{j}$. The information was modulated on the spectral amplitudes $b_{1}$ and $b_{2}$ using Gray-mapped multi-ring constellations with 4 rings and 4 uniformly spaced phases per ring $\left(0, \frac{\pi}{2}, \pi, \frac{3 \pi}{2}\right)$. The ring amplitudes were heuristically chosen to be in geometric progression with the values in Table 2. The maximum pulse duration (99.975\% energy) was 8.0535 in normalized units. The NLSE normalization factor was $T / t=8.278 \cdot 10^{-11}$. The transmission rate was $1.5 \mathrm{Gbaud}$, or $12 \mathrm{Gbps}$, and the bandwidth was $58.9 \mathrm{GHz}$. To account for the effects of inter-symbol interference (ISI), we simulated the propagation in blocks of 64 modulated pulses.
Table 2 Ring amplitudes of the constellations on $b_{1}$ and $b_{2}$

\begin{tabular}{l|llll}
\hline$b_{1}$ & 0.0163 & 0.2534 & 3.9462 & 61.4517 \\
\hline$b_{2}$ & 0.0642 & 0.4004 & 2.4972 & 15.5724 \\
\hline
\end{tabular}

At the receiver, after band-pass filtering a forward NFT was performed using forward-backward iterations with the trapezoidal rule [12] to compute the received spectral amplitudes $y_{k}[n]$. The $y_{k}[n]$ were mapped to the constellation points using (a) minimum Euclidean distance (MD) or (b) our EM algorithm. For the EM algorithm, we used different training set sizes $N$ between 6912 and 25600 symbols (note that the method is blind and the 'training' set is used to transmit data). After training, the EM parameters $\Xi$ were kept fixed and further tested on new symbols. The total number of symbols (training plus testing) was 90112 in all cases. The bit error rate (BER) results are plotted in Fig. 3 as a function of link length (with span length of $\mathcal{L}_{S}=25 \mathrm{~km}$ ). As expected, the performance of EM depends on the size of the training set, $N$. We have observed that different symbols become unreliable at different distances. This often results in 'staircase-like' BER curves, which could explain the seemingly saturating BER curves in Fig. 3. At BER $=10^{-3}$, with a training set size of 16128 symbols, the reach of EM is $30 \mathrm{~km}$ larger than that of MD. At about 20000 training symbols, the performance of EM saturates at $60 \mathrm{~km}$ larger reach than MD.

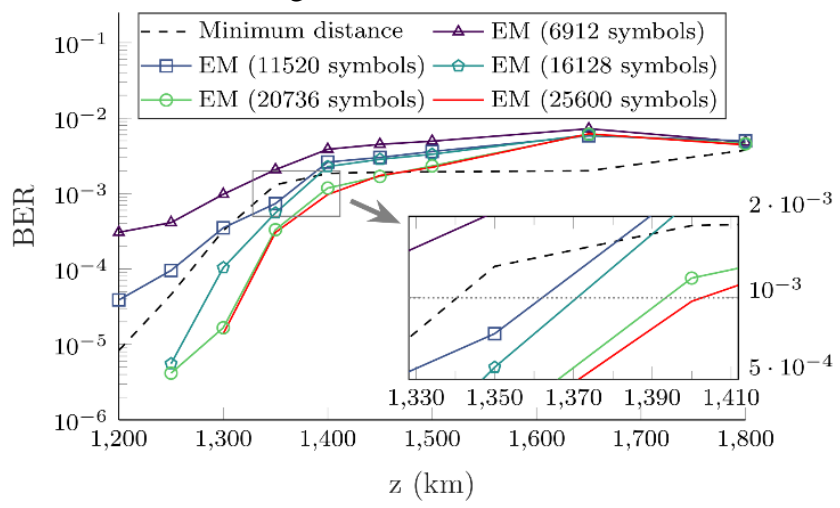

Fig. 3: Bit error rate for minimum distance (dashed) and EM (solid) detection for different training set sizes.

\section{Conclusion}

We have shown that the transmission distance of an optical transmission system with signal modulation in the NFT domain over Raman amplified fiber can be increased by utilizing the EM-algorithm to cluster the received discrete spectral amplitudes. Even though the described system is not able to compete with linearly modulated transmission regarding its spectral efficiency, it can improve transmission via the NFT, which is seen as a promising candidate to overcome the nonlinearity limit in the high input power regime of state-of-the-art optical transmission systems.

\section{Acknowledgement}

The authors wish to thank Prof. Gerhard Kramer for useful comments and careful reading of the manuscript. 


\section{References}

[1] R.-J. Essiambre, G. Kramer and P. Winzer et al, "Capacity Limits of Optical Fiber Networks," Journal of Lightwave Technology, vol. 28, no. 4, pp. 662-701, 2010 .

[2] M. I. Yousefi and F. R. Kschischang, "Information Transmission Using the Nonlinear Fourier Transform, Part I-III," IEEE Trans. Inf. Theory, vol. 60, no. 7, pp. 4312-4328, 2014.

[3] M. Ablowitz and H. Segur, Solitons and the Inverse Scattering Transform, Society for Industrial and Applied Mathematics, 1981.

[4] D. J. Kaup, "A Perturbation Expansion for the Zakharov-Shabat Inverse Scattering Transform," SIAM Journal on Applied Mathematics, vol. 31, no. 1, pp. 121-133, 1976.

[5] J. P. Gordon and H. A. Haus, "Random walk of coherently amplified solitons in optical fiber transmission," Optics Letters, vol. 11, no. 10, pp. 665667, 1986.

[6] S. Wahls, "Second Order Statistics of the Scattering Vector Defining the D-T Nonlinear Fourier Transform," in Int. ITG Conf. Systems, Commun. and Coding (SCC), Hamburg, 2017.

[7] A. P. Dempster, N. M. Laird and D. B. Rubin, "Maximum Llikelihood from incomplete data via the EM algorithm," Journal of the Royal Statistical Society: Series B (Methodological), 1977.

[8] D. Zibar, O. Winther and N. Franceschi et al, "Nonlinear Impairment Compensation Using Expectation Maximization for Dispersion Managed and Unmanaged PDM 16-QAM Transmission," Opt. Express, vol. 20, no. 26, pp. B181-B196, 2012.

[9] N. J. Muga, M. C. Fugihara and M. F. S. Ferreira et al, "Non-Gaussian ASE Noise in Raman Amplification Systems," Journal of Lightwave Technology, vol. 27, no. 16, pp. 3389-3398, 2009.
[10] Q. Lin and G. P. Agrawal, "Raman Response Function for Silica Fibers," Optics letters, vol. 31, no. 21, pp. 3086-3088, 2006.

[11] H. W. Kuhn, "The Hungarian Method for the Assignment Problem," Naval Research Logistics Quarterly, vol. 2, no. 1-2, pp. 83-97, 1955.

[12] V. Aref, "Control and Detection of Discrete Spectral Amplitudes in Nonlinear Fourier Spectrum," arXiv, 2016. 